\title{
ANÁLISE DO DESENVOLVIMENTO MOTOR DE BEBÊS PRÉ-TERMO
}

Siméia Gaspar Palácio, Ildane Oliveira dos Santos e Thamíris Rita Vieira Guimarães

UniCesumar.

simeia.palacio@unicesumar.edu.br

Acesso DOI: http://dx.doi.org/10.34059/ciejop.2019v27i1-6

\section{RESUMO}

A prematuridade predispõe o neonato a maiores riscos de comorbidades, sendo uma delas o atraso no desenvolvimento motor devido à imaturidade dos órgãos e sistemas. Desta forma, esse estudo teve como objetivo avaliar o desempenho motor de bebês prematuros. Foram incluídos no projeto, prematuros com idade gestacional $\leq 37$ semanas e excluídos neonatos a termo com doenças e síndromes neurológicas associadas. Os bebês foram triados das Unidades Básicas de Saúde e avaliados através da escala Alberta motor Infant Scale. Após a análise do desenvolvimento foi constatado que $72,72 \%$ dos bebês apresentaram desenvolvimento motor normal, enquanto $27,28 \%$ apresentaram risco de atraso no desenvolvimento.

PALAVRAS-CHAVE: prematuro, desempenho motor, fisioterapia.

\section{ABSTRACT}

Prematurity predisposes the neonate to greater risks of comorbidities, one of which is the delay in motor development due to the immaturity of the organs and systems. Thus, this study aimed to evaluate the motor performance of preterm infants. Preterm infants with gestational age $\leq 37$ weeks and excluded full-term infants with associated neurological diseases and syndromes were included in the study. The infants were screened from the Basic Health Units and evaluated through the Alberta motor Infant scale scale (AIMS). After the analysis of the development, $72,72 \%$ of the babies had normal motor development, while $27,28 \%$ presented suspicious motor performance.

KEYWORDS: premature, motor performance, physiotherapy

\section{INTRODUÇÃO}

A prematuridade é classificada de acordo com a idade gestacional ao nascimento, sendo considerado o bebê termo aquele que nasce com idade gestacional de 37 a 42 semanas e o pré-termo o recém-nascido com idade gestacional inferior a 37 semanas (SILVA, 2017). 
Dentre as causas da prematuridade merecem destaque a má formação uterina, o baixo nível socioeconômico, gestações múltiplas, tabagismo e alterações útero-placentárias. O risco de morbimortalidade para estes bebês é tanto maior quanto menor a idade gestacional e o peso ao nascimento, havendo riscos para a adaptação à vida extrauterina devido à imaturidade dos órgãos e sistemas (PINTO et al., 2008). Este é um fator que irá comprometer o desenvolvimento da criança como um todo, desde o cognitivo até o desempenho motor e, por isso, torna-se imprescindível a atuação da fisioterapia no acompanhamento do desenvolvimento motor de bebês pré-termo (FORMIGA et al., 2009).

A detecção de anormalidades precoces visa dar oportunidades de desenvolvimento a estes bebês, já que possibilita a introdução de práticas terapêuticas que auxiliem estas crianças a minimizar possíveis atrasos no seu desenvolvimento, fazendo com que as mesmas tenham perfil compatível a de bebês termo, prevenindo a aprendizagem de padrões atípicos de movimento e postura (MATTOS, 2010). Desta forma, o acompanhamento do desenvolvimento motor visa diagnosticar alterações e dar oportunidade para o bebê prematuro se desenvolver normalmente, ajudando na sua organização global, na orientação aos pais, na humanização do ambiente, além de possibilitar movimento e a percepção adequada à idade gestacional (SILVA, 2017).

Baseando-se no pressuposto que bebês prematuros estão sujeitos a apresentarem um comprometimento em seu desenvolvimento motor quando comparados aos bebês a termo, este estudo teve como objetivo analisar 0 desempenho motor de bebês prematuros (LINHARES, 2000).

\section{METODOLOGIA}

O presente estudo foi aprovado pelo Comitê de Ética da Unicesumar- Centro Universitário de Maringá sob o parecer de número 2.904.022.

O estudo foi do tipo transversal e observacional, teve início após aprovação pelo Comitê de Ética e Pesquisa do Unicesumar-Centro Universitário de Maringá.

A pesquisa foi realizada na clínica escola da referida instituição de ensino com a participação de onze prematuros com idade cronológica entre um mês a um 
ano. Os bebês prematuros foram triados nas unidades básicas de saúde do município de Maringá-PR. Foram excluídos do estudo neonatos com lesões cerebrais, malformações congênitas e outras síndromes associadas.

Após a assinatura dos responsáveis do Termo de Consentimento Livre e esclarecido, os pais responderam uma ficha de anamnese contendo perguntas sobre a gestação, condições socioeconômicas, formação acadêmica, possível intercorrência materna durante o período gestacional e complicações neonatais. Logo após os bebês foram submetidos a uma avaliação inicial elaborada pelos pesquisadores, tendo como parâmetro a idade gestacional corrigida. Para a análise do desenvolvimento motor foi utilizada a escala Alberta Infant motor scale (AIMS).

Antes da avaliação iniciar os pesquisadores orientaram os pais a deixar os bebês com roupas leves para não interferir na qualidade dos movimentos realizados durante o teste.

O ambiente escolhido para receber os prematuros durante a análise do desenvolvimento foi uma sala disponibilizada para o atendimento do referido projeto. O ambiente em questão era calmo, silencioso e confortável para tranquilizar os bebês e os pais durante a observação.

Para a realização da análise do desenvolvimento motor os prematuros foram posicionados sobre um tablado na posição inicial de decúbito dorsal, em seguida observados por um período de tempo dando-lhes a oportunidade de movimentos espontâneos e esperados de acordo com a sua idade corrigida.

A escala Alberta Infant Motor Scale (AIMS) é um instrumento de observação, por se mostrar um instrumento dinâmico, de baixo custo e eficaz nos resultados, sendo utilizada em pesquisas tanto no meio nacional quanto internacional. A mesma foi desenvolvida no Canadá pelos fisioterapeutas Piper e Darrah com o objetivo de avaliar o desenvolvimento motor dos recém-nascidos a termo e pré-termo, a partir de 38 semanas de idade gestacional até 18 meses de idade corrigida (PIPER \& DARRAH, 1994).

A Alberta Infant Motor Scale (AIMS) é composta por 58 itens agrupados em quatro subescalas que descrevem o desenvolvimento da movimentação espontânea e de habilidades motoras em quatro posições básicas realizadas pelos prematuros 
durante o teste. As posturas observadas pelos avaliadores são prono (21 itens), supino (9 itens), sentado (12 itens) e em pé (16 itens) e os resultados são obtidos através da análise dos movimentos espontâneos da criança nas diferentes posições, observando assim a superfície do corpo que sustenta o peso, postura e movimentos antigravitacionais (PIPER et al., 1992).

Cada movimento observado durante a análise obteve escore 01 (um) e cada item não observado recebeu escore 0 (zero). Os itens observados em cada uma das subescalas são somados resultando em quatro subtotais onde o escore total (0-58 pontos) é obtido através das somas destes subtotais. A escala é composta por um gráfico constituído por dois eixos, o eixo $\mathrm{Y}$ corresponde à idade corrigida enquanto $\mathrm{O}$ eixo $X$ representa a pontuação obtida na AIMS.

Os resultados foram convertidos em percentil de desenvolvimento motor, de acordo com os seguintes critérios de classificação: 1) desempenho motor normal/esperado: acima de $25 \%$ da curva percentílica; 2) desempenho motor suspeito: entre $5 \%$ e $25 \%$ da curva percentílica; 3) desempenho motor anormal: abaixo de $5 \%$ da curva percentílica. (PIPER et al., 1992).

\section{RESULTADOS}

Quanto ao perfil da amostra investigada foram avaliados onze bebês prematuros, sendo $54,54 \%$ do gênero masculino e $45,46 \%$ do feminino, com uma média de idade gestacional de 34 semanas, idade cronológica de 6,72 meses e média de idade corrigida de 5,4 meses.

No que se refere ao desenvolvimento motor da amostra, a análise dos resultados indicou que $72,72 \%$ dos prematuros avaliados apresentaram resultados favoráveis adquirindo o percentil $>25$ th na escala de avaliação Alberta Infant Motor Scale (AIMS), enquanto que em $27,28 \%$ dos bebês foi constatado sinais de riscos para atrasos motores com um percentil variando entre 5 e 25th.

Em relação ao tipo de parto, foi verificado que houve predomínio do parto cesáreo com o resultado de $90,91 \%$ em relação ao parto normal $(9,09 \%)$. Durante o preenchimento da ficha de anamnese foi observado que $63,63 \%$ das mães 
apresentaram intercorrências durante o período gestacional, as complicações mais comumente observadas foram pressão alta, alterações útero-placentárias e diabetes gestacional.

No que diz respeito às internações após o nascimento, foi percebido que $63,63 \%$ dos prematuros apresentaram a necessidade de permanecer hospitalizados na Unidade de Terapia Intensiva (UTI) com uma média de 7,81 dias de internação, tendo como uma das causas principais a necessidade de suporte ventilatório, sendo a média de peso ao nascer dos bebês participantes do projeto foi de $2.380 \mathrm{~kg}$.

Em relação à escolaridade das mães $27,27 \%$ delas apresentaram ensino superior incompleto, $45,46 \%$ concluíram o segundo grau e $27,27 \%$ o ensino superior completo.

No referente ao perfil familiar $72,72 \%$ das mães eram casadas, com vidas conjugais estáveis, sendo a porcentagem de mães solteiras relativamente baixa com apenas $27,28 \%$ do resultado. Todas as famílias que participaram do estudo relataram que além da ajuda de seus parceiros eram constantemente auxiliadas pelas avós das crianças.

\section{CONCLUSÃO}

Levando em consideração os aspectos mencionados no referido estudo, observou-se que a maioria dos bebês apresentaram desenvolvimento motor compatível com a idade gestacional corrigida. Porém devido a escassez da amostra sugere-se que novos estudos sejam realizados em âmbito nacional que investiguem sobre análises do desenvolvimento motor em prematuros, tendo como um dos parâmetros as peculiaridades entre os gêneros feminino e masculino, ou ainda estudos que visem comparar as diferenças do desenvolvimento motor em prematuros gemelares.

\section{REFERÊNCIAS}

ALMEIDA, Adriana Carvalho de; JESUS, Ana Cristina Pereira de; LIMA, Pamylla Fortes Tanikawa; ARAÚJO, Márcio Flávio Moura de; ARAÚJO, Thiago Moura de. 
Fatores de risco maternos para prematuridade em uma maternidade pública de imperatriz-Ma. Imperatriz-MA. Revista Gaúcha de Enfermagem, v. 33 n. 2. p. 8694, Jun 2012.

CAÇOLA, Priscila; BOBBIO, Tatiana Godoy. Baixo peso ao nascer e alterações no desenvolvimento motor: a realidade atual. Revista Paulista de Pediatria, v. 28 n.1 p.70-6, 2010.

CASCAES, Andreia Morales; GAUCHE, Heide; BARAMARCHI, Fabiana Martin; BORGES, Carolina Marques; PERES, Karen Glazer. Prematuridade e fatores associados no Estado de Santa Catarina, Brasil, no ano de 2005: análise dos dados do Sistema de Informações sobre Nascidos Vivos. Cadernos de Saúde Pública, v. 24 n.5 p. 1024-1032, Mai 2008.

COVARRUBIAS, Lorenzo Osorno, AGUIRRE, Gladys Esther Rupay, CHAPUZ, José Rodríguez; MAY, Ana Isabel Lavadores; VELÁZQUEZ, Jorge Dávila; EGUILUZ, Manuel Echeverría. Factores maternos relacionados con prematuridad. Ginecol Obstet Mex, v. 76, n. 9, p. 526-536, 2008.

FORMIGA, Cibelle Kayenne Martins Roberto; LINHARES, Maria Beatriz Martins. Avaliação do desenvolvimento inicial de crianças nascidas pré-termo. Revista da Escola de Enfermagem da USP, v.43 n. 2 p. 472-480, 2009.

FRIGO, Jucimar; ZOCCHE, Denise Antunes de Azambuja; PALAVRO, Gislaine Laiz; TURATTI, Leticia Aparecida; NEVES, Eliane Tatsch; SCHAEFER, Tania Mariga. Percepções de pais de recém-nascidos prematuros em unidade de terapia intensiva neonatal. Revista de Enfermagem da UFSM, v. 5 n.1 p.58-68, Jan/Mar 2015.

GUIMARÃES, Elaine da Costa; MELO, Enirtes Caetano Prates. Características do apoio social associados à prematuridade em uma população de puérperas de baixa renda. Escola Anna Nery Revista de Enfermagem, v.15 n.1 p. 54-61, Jan/Mar 2011.

JÚNIOR, Mário Dias Corrêa; FONSECA, Eduardo Sérgio Valério Borges da; CALISTO, Aline Costa; EVANGELISTA, Aline Arão. Predição e prevenção do parto pré-termo espontâneo. Revista Médica de Minas Gerais, v. 23 n.3 p.330-335, Jul 2013.

LINHARES, Maria Beatriz Martins; VITA, Ana Emilia Carvalho; BORDIN, Maria Beatriz Machado; CHIMELLO, Juliana Thomazatti; MARTINEZ, Francisco Eulógio; JORGE, Salim Moysés. Prematuridade e muito baixo peso como fatores de risco ao desenvolvimento da criança. Paidéia (Ribeirão Preto),v. 10 n.18 p. 60-69, Jan/Jul 2000.

MAIA, Polyana Candeia; SILVA, Larissa Paiva; OLIVEIRA, Márcia Maria Coelho; CARDOSO, Maria Vera Lúcia Moreira Leitão. Desenvolvimento motor de crianças prematuras e a termo: uso da Alberta Infant Motor Scale. Acta Paulista de Enfermagem, v. 24 n.5 p.670-675, Jun 2011. 
MATTOS, Bruna Marturelli; BELLANI, Claudia Diehl Forti. A importância da estimulação precoce em bebês portadores de síndrome de down: revisão de literatura. Revista brasileira de terapia e saúde, v.1 v.1, p. 51-63, jul 2010.

NAVAJAS, Andréa Felner; CANIATO, Francine. Estimulação precoce/essencial: a interação família e bebê pré-termo (prematuro). Cadernos de Pós-Graduação em Distúrbios do Desenvolvimento, v. 3 n.1 p. 59-62, 2003.

OLIVEIRA, Rosana Rosseto de; Melo, Emiliana Cristina; NOVAES, Elisiane Soares; FERRACIOLI, Patrícia Louise Rodrigues Varela; MATHIAS, Thais Aidar de Freitas. Fatores associados ao parto cesárea nos sistemas público e privado de atenção à saúde. Revista da Escola de Enfermagem da USP, v. 50, n. 5, p. 734-741, jul.2016.

PINTO, Mariana; SILVA, Claudia Ferreira Gomes da; MUNARI, Maitê Marques; ALMEIDA, Carla Skilhan de; RESENDE, Thaís de Lima. Intervenção motora precoce em neonatos prematuros. Revista da Graduação Publicações de TCC, v.1, n. 2, p.1-10, 2008.

PIPER M.C, Pinnell LE, Darrah J, Maguire T, Byrne PJ. Construction and validation of the Alberta Infant Motor Scale (AIMS). Can J Public Health, v. 83, n. 2, p. 46-50, jul-ago.1992.

PIPER, M.C.; Darrah, J. Motor assessment of the developing infant. Ed. 1. Philadelphia: Saunders Company,1994.

RAMOS, Helena Ângela de Camargo; CUMAN, Roberto Kenji Nakamura. Fatores de risco para prematuridade: pesquisa documental. Esc Anna Nery Rev Enferm, v. 13, n. 2, p. 297-304, abr-jun.2009.

SALGE, Ana Karina Marques; VIEIRA, Aline Vaz da Costa; AGUIAR, Anne Kelly Araújo; LOBO, Sara Fleury; XAVIER, Raphaela Maioni; ZATTA, Laidilce Teles; CORREA, Rosana Rosa Miranda; SIQUEIRA, Karina Machado; GUIMARÃES, Janaína Valadares; ROCHA, Karlla Morgana Nunes; CHINEM, Brunella Mendonça; Silva, Renata Calciolari Rossi e. Fatores maternos e neonatais associados à prematuridade. Revista Eletrônica de Enfermagem, v.11, n. 3, p. 642-646,set. 2009.

SAMPAIO, Rafaella Maria Monteiro; PINTO, Francisco José Maia; SAMPAIO, Josiane do Carmo. Fatores de risco associados à prematuridade em nascidos vivos no estado do Ceará. Revista Baiana de Saúde Pública, v. 36, n. 4, p. 969-978, 2013.

SILVA, Carla Cavalcante Ventura. Atuação da fisioterapia através da estimulação precoce em bebês prematuros. Rev. Eletrôn. Atualiza Saúde, Salvador, v. 5, n. 5, p. 29-36, jan./jun. 2017.

SILVA, Cristiane Alves; BRUSAMARELLO, Sheila; CARDOSO, Fernanda Guimarães C.; ADAMCZYK, Natasha Freixiela; NETO, Francisco Rosa. Desenvolvimento de 
prematuros com baixo peso ao nascer nos primeiros dois anos de vida. Rev Paul Pediatr, v. 29, n. 3, p. 328-335, jan. 2011

SILVEIRA, Mariângela F; VICTORA, Cesar G.; BARROS, Aluísio J D.; SANTOS, Iná S.; MATIJASEVICH, Alicia; BARROS, Fernando C. Determinantes de nascimento pré-termo na coorte de nascimentos de 2004, Pelotas, Rio Grande do Sul, Brasil. Cadernos de saúde pública/Ministério da Saúde, Fundação Oswaldo Cruz, Escola Nacional de Saúde Pública., v. 26, n. 1, p. 185-194, jan.2010. 